\title{
Epidemiology of hepatocellular carcinoma in metabolic liver disease
}

\author{
Stefano Bellentani \\ Consultant Gastroenterologist and Hepatologist 6600 Locarno, Ticino, Switzerland.
}

Correspondence to: Dr. Stefano Bellentani, Studio Medico - Via Della Pace 3, 6600 Locarno, Ticino, Switzerland. E-mail: bellentanistefano@gmail.com

How to cite this article: Bellentani S. Epidemiology of hepatocellular carcinoma in metabolic liver disease. Hepatoma Res 2020;6:29. http://dx.doi.org/10.20517/2394-5079.2020.10

Received: 3 Feb 2020 First Decision: 10 Apr 2020 Revised: 16 Apr 2020 Accepted: 20 Apr 2020 Published: 16 Jun 2020

Science Editor: Darrell Crawford Copy Editor: Jing-Wen Zhang Production Editor: Jing Yu

\begin{abstract}
Nonalcoholic fatty liver disease and its evolutive form nonalcoholic steatohepatitis (NASH) are nowadays the second/third cause of chronic liver disease worldwide, and their prevalence and incidence are rapidly increasing in parallel to the burden of diabetes and obesity. Hepatocellular carcinoma (HCC) due to NASH (HCC-NASH) has become the major cause of HCC and is now one of the major indications for liver transplant in Western countries, after that due to HCV infection. NASH occurs both in the presence and absence of liver cirrhosis. In this review, we describe the epidemiology of HCC related to metabolic liver disease: not only NASH-HCC but also type 2 diabetes mellitus and obesity-related HCC. Some new practical guidelines for screening and surveillance of patients with metabolic diseases at risk for HCC are also discussed.
\end{abstract}

Keywords: Nonalcoholic steatohepatitis, hepatocellular carcinoma, metabolic syndrome, obesity, type 2 diabetes mellitus

\section{INTRODUCTION}

The prevalence of nonalcoholic steatohepatitis (NASH)-related hepatocellular carcinoma (HCC) is increasing in parallel with the prevalence of nonalcoholic fatty liver disease (NAFLD), type 2 diabetes mellitus (T2DM) and obesity worldwide, particularly in Western countries ${ }^{[1]}$. HCC develops in patients with NASH and without cirrhosis in $40 \%$ of cases $^{[2,3]}$. In patients with NASH and advanced (F3) fibrosis or Child A cirrhosis, approximately $20 \%$ of patients progress to decompensated cirrhosis over a 2-year

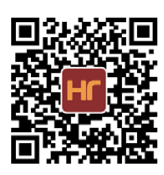


period $^{[4,5]}$. There are significant geographic variations in the incidence of NASH and NASH-HCC ${ }^{[6,7]}$. HCC, in general, has the sixth highest cancer incidence, and is the fourth leading cause of cancer-related death in the world ${ }^{[8]}$. It is already known that the most prevalent cancer in obesity and T2DM is HCC, and that the prevalence of NAFLD/NASH in obesity and T2DM is significantly higher than in the general population, being $75 \%, 95 \%$ and $25 \%$, respectively ${ }^{[9,10]}$. Obesity and high-fat diet are increasingly recognized as major risk factors for HCC-NASH, but the exact molecular mechanisms integrating these events remain unclear $^{[11]}$. Furthermore, the etiology and pathogenic link between obesity, T2DM and NASH-HCC is not completely understood, at least in humans. It could be related to STAT-1/STAT-3 intracellular signaling ${ }^{[12]}$ or, according to new pathogenetic interpretation, also to alteration of the gut microbiota ${ }^{[11]}$. We also know that a substantial proportion of patients with NAFLD/NASH will never develop HCC, and thus, it is very important to understand the exact relationship between NASH-cirrhosis and NASH-HCC and metabolic diseases to program good prevention and treatment measures for these patients.

We try to unravel these still less known diseases in the following section.

\section{THE NATURAL HISTORY OF NASH}

NAFLD is still interpreted as a negative definition used to describe a spectrum of metabolic liver diseases due to excessive hepatic fat accumulation with associated insulin resistance, in the absence of significant alcohol consumption or other causes ${ }^{[13,14]}$. While NAFLD can refer to simple steatosis, NASH is characterized by inflammation and is associated with an increased risk of progression to fibrosis, advanced fibrosis, cirrhosis, hepatic decompensation and $\mathrm{HCC}^{[13,14]}$. NASH can only be reliably differentiated from NAFLD histologically, and the prevalence of NASH among biopsied NAFLD patients is estimated to be $59.1 \%$ (95\%CI: $47.55 \%-69.73 \%$ ) from pooled NASH prevalence data ${ }^{[15]}$. Fibrosis is an important bad prognostic factor in NAFLD/NASH and in patients with NASH and advanced (F3) fibrosis or compensated cirrhosis, and it has been shown that approximately $20 \%$ of them will progress to cirrhosis/HCC, or hepatic decompensation over a 2-year period ${ }^{[4,5]}$. As shown in Figure 1 and as reported by Goah and McCollough ${ }^{[5]}$, the natural history of NASH and its passage to advanced fibrosis, and then to cirrhosis and to HCC, depends on different risk factors such as sex, age, obesity, T2DM, genetic factors, and fibrosis stage. The first steps are also driven by a genetic predisposition contributing by itself to the development of insulin resistance and hepatic steatosis. Other co-factors could determine the lower or higher rate of progression from NASH to advanced fibrosis and to cirrhosis or HCC [Figure 1].

It must also be considered that NAFLD and NASH prevalence and incidence are different according to sex, usually higher in men than in premenopausal women (or age $\leq 50-60$ years), while they tend to become more common in women after menopause (or age $\geq 50-60$ years) ${ }^{[16]}$. This is probably due to sex hormone levels that might influence hepatic lipid and carbohydrate metabolism; particularly, it seems that estrogens may protect the liver from fibrosis in NAFLD/NASH. Estrogen seems to protect also from liver tumorigenesis, and theoretically, prolonging estrogen depletion (i.e., premature menopause) may lead to increased HCC risk in women with NASH ${ }^{[16]}$.

\section{OBESITY, GUT MICROBIOTA AND NASH-HCC}

\section{Mechanisms of obesity induced HCC}

Obesity is a major driver of cancer, especially HCC.

The prevailing view is that NASH and fibrosis or cirrhosis are required for HCC in obesity. However, they can also be dissociated in obesity. A recent work by Grohmann ${ }^{[1]]}$ in mice showed a strict correlation between STAT-1 signaling in inducing NASH and fibrosis and STAT-3 in promoting HCC formation. The authors were able also to demonstrate that STAT-3 signaling can drive tumor development in a context 


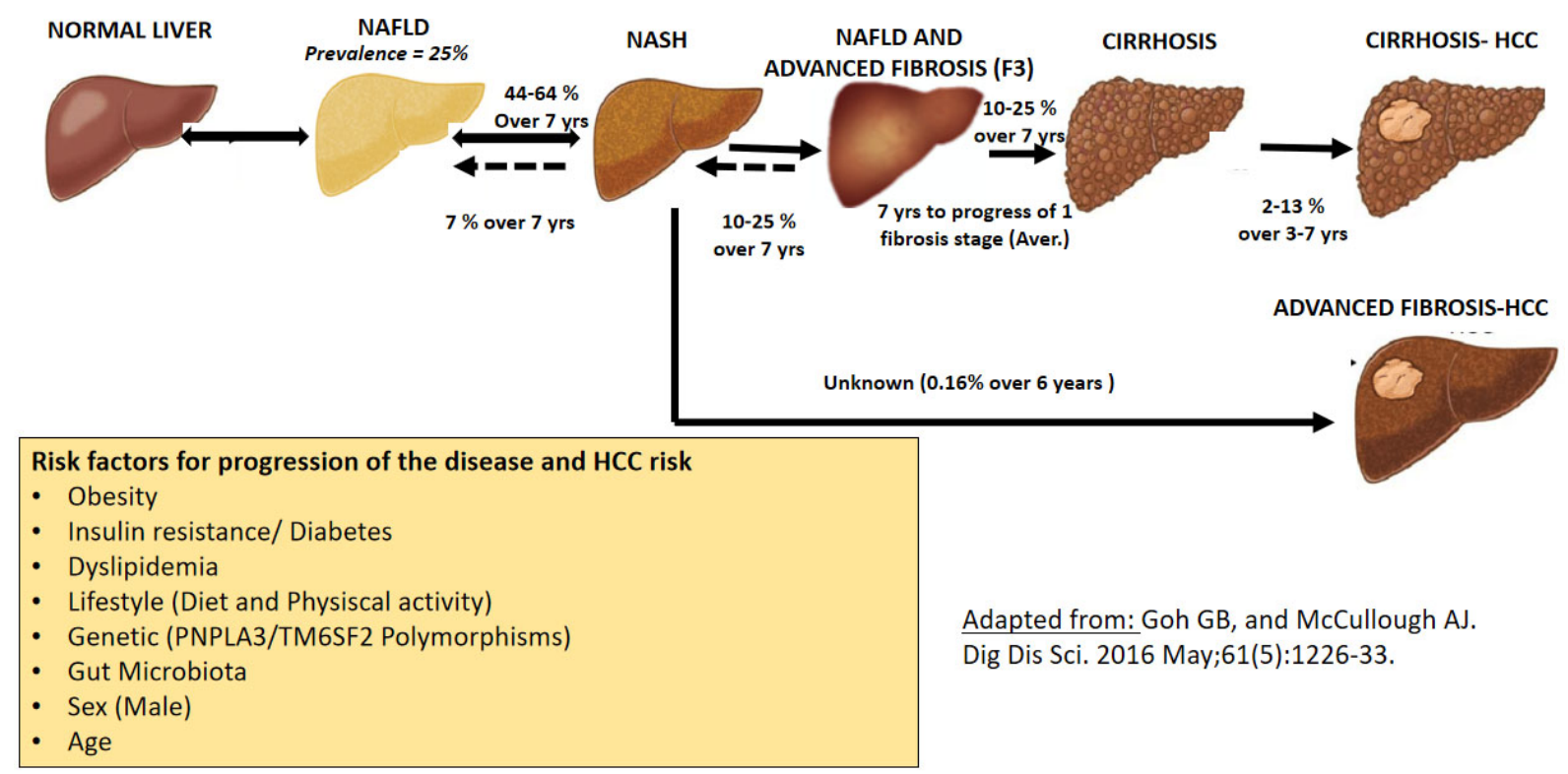

Figure 1. Natural history of NASH and risk factors for progression of NASH to HCC. Adapted from study ${ }^{[5]}$. NASH: nonalcoholic steatohepatitis; HCC: hepatocellular carcinoma; NAFLD: nonalcoholic fatty liver disease

of obesity with NAFLD, independently of the presence of NASH and fibrosis. Their studies reveal how obesity-associated hepatic oxidative stress can independently contribute to the pathogenesis of NASH, fibrosis and HCC, but again in mice.

Furthermore, HCC exhibits several characteristic lipid metabolic changes, especially in fatty acid (FA) metabolism, which is linked to NASH and associated with the status of other metabolic pathways, such as glucose metabolism.

Accumulating evidence supports the importance of lipid metabolic reprogramming in various situations of hepatocarcinogenesis. In a recent review, the latest findings regarding the role of FA metabolism pathways in the development of HCC, focusing on reprogramming lipid metabolism, have been discussed in $\operatorname{depth}^{[17]}$.

Another recent study reported ${ }^{[18]}$ that unconventional prefoldin RPB5 interactor (URI) induces DNA damage in hepatocytes and triggers inflammation via T helper 17 (Th17) lymphocytes and interleukin-17A (IL-17A). This induces neutrophil infiltration into white adipose tissue, mediating insulin resistance (IR) and FA release, stored in liver as triglycerides, causing NASH and subsequently NASH-HCC, suggesting that IL-17A blockers may prevent IR, NASH, and HCC in high-risk patients.

\section{Mechanisms of gut microbiota-induced obesity and HCC}

Alterations of intestinal microbiota seem to play a key role in this pathogenetic mechanism. Yoshimoto et al. ${ }^{[19]}$ studied hepatocarcinogenesis in obese mice, showing that the administration of antibiotics and gut sterilization could decrease the development of HCC in treated mice, modulating the dysbiosis and the subsequent secretion of pro-inflammatory and pro-carcinogenetic factors. Their data suggested that gut sterilization and antibiotic treatments could prevent the development of HCC, but these treatments did not lead to the regression of already established tumors. Several other lines of research showed that the gut microbiota is also involved in the development of HCC, in particular by increasing lipopolysaccharide levels and creating a subsequent pro-inflammatory microenvironment in the liver. Another mechanism 
could be the one discovered recently by Loo et al. ${ }^{[20]}$ involving lipoteichoic acid, a gram-positive gut microbial component that seems to promote HCC development by creating a tumor-promoting microenvironment. Lipoteichoic acid enhances the senescence-associated secretory phenotype of hepatic stellate cells (HSC) collaboratively with the obesity-induced gut microbial metabolite deoxycholic acid to upregulate the expression of senescence-associated secretory phenotype factors and COX2 through Tolllike receptor 2. Interestingly, COX2-mediated prostaglandin E2 (PGE2) production suppresses antitumor immunity through a PTGER4 receptor, thereby contributing to HCC progression.

Moreover, COX2 overexpression and excess PGE2 production have been detected in HSCs in patients with NASH-HCC. The gut microbiota-driven COX2 pathway produces the lipid mediator PGE2 in senescent HSCs, which plays a pivotal role in suppressing antitumor immunity, suggesting that also PGE2 and its receptor may be novel therapeutic targets for noncirrhotic HCC-NASH ${ }^{[18]}$.

All these new experimental findings discovering links between lipid metabolism, inflammation, insulin resistance, gut microbiome and HCC-NASH are still to be confirmed in humans, but they demonstrate that the pathogenetic pathway of NASH-HCC is multifactorial, very complex and still far away from leading to an efficacious treatment for this increasingly prevalent cancer.

\section{T2DM AND NASH-HCC}

T2DM was also found to be an independent factor associated with HCC among patients with cryptogenic NASH-cirrhosis since $2002^{[21]}$. However, the association between diabetes and the risk of HCC in NASH patients with cirrhosis is not well quantified and completely understood. Diabetes increases the risk of liver disease progression to cirrhosis in patients with NASH. Only recently, Yang et al. ${ }^{[22]}$ investigated the association between diabetes and HCC in patients with NASH cirrhosis in an large cohort with a longer follow-up and were able to demonstrate, even also in humans, that T2DM is associated with an increased risk of HCC in patients with NASH cirrhosis. Their secondary aim was to investigate the association between other metabolic risk factors (body mass index, hypertension, and hyperlipidemia) and HCC. However, the above metabolic risk factors were not associated with HCC risk $^{[22]}$.

Unfortunately there are not many other studies in humans that help to elucidate the molecular mechanisms underlying the pathogenesis of NASH-HCC related to T2DM. Progress in this field depends on the availability of reliable preclinical models amenable to genetic and functional analyses and exhibiting robust NASH-to-HCC progression.

\section{GENETIC, LIVER METABOLIC DISEASE AND NASH-HCC}

Only recently was the association between NASH and genetic predisposition investigated, especially in young adults with NASH without any metabolic diseases. The most recent and important study is the one by Unalp-Arida and Ruhl ${ }^{[21]}$. They examined the relationships of liver disease markers, including patatinlike phospholipase domain-containing protein 3 (PNPLA3) I148M,w ith mortality in a very large series (13,298 viral hepatitis-negative adults) belonging to the United States National Health and Nutrition Examination Survey, 1988-1994, with 27 years of linked mortality data. They found that PNPLA3 I148M was associated with increased liver disease mortality, and they suggested that the genetic variant PNPLA3 I148M may be used as a marker in patients with NAFLD/NASH for HCC surveillance.

\section{SCREENING AND SURVEILLANCE OF HCC IN PATIENTS WITH NASH-CIRRHOSIS OR METABOLIC DISEASES}

There is now substantial evidence that NAFLD/NASH-associated HCC may arise either in the presence or absence of cirrhosis ${ }^{[3,4,13,14,22-24]}$. Limited data are available to address the clinician's need to know who is 
the NASH patient or patients with metabolic disease who should undergo screening and surveillance for HCC, and many questions remain to be answered, including new and efficacious strategies for targeting high-risk subjects in the general population ${ }^{[25]}$. A recent evidence-based expert review ${ }^{[24]}$ tries to clarify this matter, and summarizes the American Gastroenterology Association practical update on screening and surveillance for HCC in patients with NAFLD/NASH ${ }^{[24]}$. In this review, different practical guidelines are suggested for 3 groups of patients: 1- patients with NASFLD/NASH and without advanced fibrosis; 2- patients with advanced fibrosis, and finally, 3- patients with NASH-cirrhosis ${ }^{[24]}$. In the first group, based on a recent national Veterans Affairs study in the USA, HCC incidence rates of patients with NASFLD/ NASH and not advanced fibrosis were $0.21 / 1000$ person-years (0.02\% annual risk) in NAFLD and $0.02 / 1000$ person-years $(0.002 \%$ annual $)$ in controls ${ }^{[26,27]}$. Although there was a higher risk of developing HCC in patients with earlier stages of NAFLD and low fibrosis than in those without NAFLD, the authors concluded that the risk estimate is likely to be too low to justify routine screening in those who have early NAFLD with no evidence of advanced fibrosis ${ }^{[2]}$.

Considering the second group, HCC screening in patients who have NAFLD/NASH with advanced fibrosis, as determined by combining at least 2 noninvasive score testing modalities suggestive of cirrhosis (in the absence of biopsy-confirmed cirrhosis or overt cirrhosis on imaging), associated with diagnosis of cirrhosis at elastography examination (i.e., with fibroscan or share-wave elastography, or quantitative MRI), patients in whom both tests are concordant for advanced fibrosis or cirrhosis should be, on the contrary, considered for HCC screening ${ }^{[2]]}$. Finally, in the third group, the incidence rate of HCC in NAFLD cirrhosis is estimated to be $>1.5 \%$ per year, and therefore, screening for HCC in this group is justifiable, based on cost-effectiveness considerations. Therefore, the best practice guidance recommendation ${ }^{[24]}$ is to consider and offer HCC screening and surveillance to all patients with NAFLD/NASH compensated cirrhosis, in patients with NAFLD/NASH and advanced fibrosis, but not in patients with simple NAFLD or NASH and non-advanced fibrosis ${ }^{[24]}$.

\section{CONCLUSION}

NAFLD/NASH is an important cause of liver disease worldwide and is associated with an increased risk of developing HCC, either in the presence or absence of liver cirrhosis. The prevalence of metabolic liver disease and of NASH-HCC is increasing in parallel with the prevalence of obesity and T2DM worldwide. The future global burden of NAFLD-related HCC is going to become a major public health issue, so further research to identify the factors involved in promoting inflammation and hepatocarcinogenesis in NAFLD/ $\mathrm{NASH}$ and to program cost-effective prevention and treatment strategies are urgently needed.

\section{DECLARATIONS}

\section{Authors' contributions}

The author contributed solely to the article.

\section{Availability of data and materials}

Not applicable.

\section{Financial support and sponsorship}

None.

\section{Conflicts of interest}

The author declared that there are no conflicts of interest.

\section{Ethical approval and consent to participate}

Not applicable. 


\section{Consent for publication}

Not applicable.

\section{Copyright}

(c) The Author(s) 2020.

\section{REFERENCES}

1. Bellentani S. The epidemiology of non-alcoholic fatty liver disease. Liver Int 2017;37:81-4.

2. Bellentani S, Baroni GS, Piscaglia F, Tiribelli C. Natural history of nonalcoholic steatohepatitis-associated hepatocellular carcinoma. Clin Liver Dis (Hoboken) 2016;8:105-7.

3. Piscaglia F, Svegliati-Baroni G, Barchetti A, Pecorelli A, Marinelli S, et al. Clinical patterns of hepatocellular carcinoma in nonalcoholic fatty liver disease: a multicenter prospective study. Hepatology 2016;63:827-38.

4. Loomba R, Adams LA. The $20 \%$ rule of NASH progression: the natural history of advanced fibrosis and cirrhosis caused by NASH. Hepatology 2019;70:1885-8.

5. Goh GB, McCullough AJ. Natural history of nonalcoholic fatty liver disease. Dig Dis Sci 2016;61:1226-33.

6. Estes C, Anstee QM, Arias-Loste MT, Bantel H, Bellentani S, et al. Modeling NAFLD disease burden in China, France, Germany, Italy, Japan, Spain, United Kingdom, and United States for the period 2016-2030. J Hepatol 2018;69:896-904.

7. Goossens N, Bellentani S, Cerny A, Dufour JF, Jornayvaz FR, et al. Nonalcoholic fatty liver disease burden - Switzerland 2018-2030. Swiss Med Wkly 2019;149:w20152.

8. Fitzmaurice C, Allen C, Barber RM, Barregard L, Bhutta ZA, et al.; Global Burden of Disease Cancer Collaboration. Global, regional, and national cancer incidence, mortality, years of life lost, years lived with disability, and disability-adjusted life-years for 32 cancer groups, 1990 to 2015: a systematic analysis for the global burden of disease study. JAMA Oncol 2017;3:524-48.

9. Bellentani S, Saccoccio G, Masutti F, Giacca M, Miglioli L, et al. Risk factors for alcoholic liver disease. Addict Biol 2000;5:261-8.

10. Bedogni G, Miglioli L, Masutti F, Tiribelli C, Marchesini G, et al. Prevalence of and risk factors for nonalcoholic fatty liver disease: the Dionysos nutrition and liver study. Hepatology 2005;42:44-52.

11. Brandi G, De Lorenzo S, Candela M, Pantaleo MA, Bellentani S, et al. Microbiota, NASH, HCC and the potential role of probiotics. Carcinogenesis 2017;38:231-40.

12. Grohmann M, Wiede F, Dodd GT, Gurzov EN, Ooi GJ, et al. Obesity drives STAT-1-dependent NASH and STAT-3-dependent HCC. Cell 2018;175:1289-306.e1220.

13. European Association for the Study of the Liver (EASL); European Association for the Study of Diabetes (EASD); European Association for the Study of Obesity (EASO). EASL-EASD-EASO Clinical Practice Guidelines for the management of non-alcoholic fatty liver disease. J Hepatol 2016;64:1388-402.

14. Chalasani N, Younossi Z, Lavine JE, Charlton M, Cusi K, et al. The diagnosis and management of nonalcoholic fatty liver disease: practice guidance from the American Association for the Study of Liver Diseases. Hepatology 2018;67:328-57.

15. Younossi ZM, Koenig AB, Abdelatif D, Fazel Y, Henry L, et al. Global epidemiology of nonalcoholic fatty liver disease-Meta-analytic assessment of prevalence, incidence, and outcomes. Hepatology 2016;64:73-84.

16. Lonardo A, Nascimbeni F, Ballestri S, Fairweather D, Win S, et al. Sex differences in nonalcoholic fatty liver disease: state of the art and identification of research gaps. Hepatology 2019;70:1457-69.

17. Nakagawa H, Hayata Y, Kawamura S, Yamada T, Fujiwara N, et al. Lipid metabolic reprogramming in hepatocellular carcinoma. Cancers (Basel) 2018;10:447.

18. Gomes AL, Teijeiro A, Buren S, Tummala KS, Yilmaz M, et al. Metabolic inflammation-associated IL-17A causes non-alcoholic steatohepatitis and hepatocellular carcinoma. Cancer Cell 2016;30:161-75.

19. Yoshimoto S, Loo TM, Atarashi K, Kanda H, Sato S, et al. Obesity-induced gut microbial metabolite promotes liver cancer through senescence secretome. Nature 2013;499:97-101.

20. Loo TM, Kamachi F, Watanabe Y, Yoshimoto S, Kanda H, et al. Gut microbiota promotes obesity-associated liver cancer through PGE2mediated suppression of antitumor immunity. Cancer Discov 2017;7:522-38.

21. Bugianesi E, Leone N, Vanni E, Marchesini G, Brunello F, et al. Expanding the natural history of nonalcoholic steatohepatitis: from cryptogenic cirrhosis to hepatocellular carcinoma. Gastroenterology 2002;123:134-40.

22. Yang JD, Ahmed F, Mara KC, Addissie BD, Allen AM, et al. Diabetes is associated with increased risk of hepatocellular carcinoma in patients with cirrhosis from nonalcoholic fatty liver disease. Hepatology 2020;71:907-16.

23. Unalp-Arida A, Ruhl CE. PNPLA3 I148M and liver fat and fibrosis scores predict liver disease mortality in the United States population. Hepatology 2020;71:820-34.

24. Loomba R, Lim JK, Patton H, El-Serag HB. AGA clinical practice update on screening and surveillance for hepatocellular carcinoma in patients with nonalcoholic fatty liver disease: expert review. Gastroenterology 2020; Epub ahead of print. doi: 10.1053/j.gastro.2019.12.053

25. Younes R, Bugianesi E. Should we undertake surveillance for HCC in patients with NAFLD? J Hepatol 2018;68:326-34.

26. Patel YA, Gifford EJ, Glass LM, McNeil R, Turner MJ, et al. Risk factors for biopsy-proven advanced non-alcoholic fatty liver disease in the Veterans Health Administration. Aliment Pharmacol Ther 2018;47:268-78.

27. Reig M, Gambato M, Man NK, Roberts JP, Victor D, et al. Should patients with NAFLD/NASH be surveyed for HCC? Transplantation 2019;103:39-44. 